\title{
DAS PROBABILISTISCHE, WISSENSBASIERTE SYSTEM SPIRIT
}

\author{
Wilhelm Rödder, FernUniversität Hagen \\ Heinz Peter Reidmacher, FernUniversität Hagen
}

SPIRIT ist als wissensbasiertes System im weitesten Sinne den Expertensystemen zuzuordnen, obwohl es in seiner Konzeption und den verwendeten Methoden besondere Charakteristika aufweist, die es von herkömmlichen Entwicklungen unterscheidet. In diesem Artikel werden Konzepte, die insbesondere die Wissensrepräsentation, -akquisition, -verarbeitung und die Präsentation des Wissens in SPIRIT betreffen, vorgestellt.

Informationen, die in Form von Aussagen über eine beliebige Wissensdomäne vorliegen, werden probabilistisch durch eine Menge von Randverteilungen über Aussagengruppen repräsentiert. Die Wissensakquisition erfolgt in Abhängigkeit von statistischen Daten oder durch die Eingabe von regelbasiertem, subjektivem Expertenwissen; realisiert wird sie durch eine Bayessche Aktualisierung mit exponentieller Glättung von Wahrscheinlichkeiten der beobachteten Ereignisse. Die Strukturierung der Wissensbasis in Clustern von Aussagen erfolgt dynamisch über eingegebene Beobachtungen. Durch diesen induktiven Lernprozeß werden auch Zusammenhänge zwischen den repräsentierten Aussagen adaptiert, die Grundlage für die Strukturierung der Wissensbasis, d.h. insbesondere die Bildung der Gruppen, sind. Dadurch wird genau zwischen den Aussagen, über die starke Abhängigkeiten beobachtet wurden, auch eine reiche Informationsstruktur zur Verfügung gestellt. Da die Wissensverarbeitung entropieoptimal durchgeführt wird, sind Prognosen garantiert, die nicht durch methodisch bedingte Annahmen beeinflußt werden. Prognoseergebnisse und erlernte Informationen, die zunächst als Randverteilungen über Hypothesenmengen bzw. Aussagengruppen vorliegen, werden analysiert und als aussagekräftige wenn-dann-Regeln dargestellt. Dazu wird die Menge aller signifikanten Abhängigkeiten zwischen Aussagenmengen, die implizit in einer Wahrscheinlichkeitsverteilung enthalten sind, solange reduziert, bis mit einer möglichst kleinen Regelbasis die wichtigsten Informationen dargestellt werden können.

Abschließend werden typische Anwendungsbereiche skizziert, in denen die Vorteile von SPIRIT, insbesondere Abhängigkeiten selbständig zu erkennen, die erlernten Zusammenhänge entropieoptimal zu verarbeiten und die erlernten und prognostizierten Resultate verständlich darstellen zu können, zum tragen kommen. 